\title{
Investigation of Machining Energy in Wire Electrical Discharge Machining Using Measurement of Deform
}

\author{
Quanli Han \\ School of Mechanical \& Electrical Engineering, Xi'An Technological University, P. R. China \\ hanqljx@xatu.edu.cnail
}

Keywords: Machining energy; Electrical discharge machining; Deform

\begin{abstract}
This paper aims at investigating the machining energy in wire electrical discharge machining. The open kerf was designed on raw martial block to obverse deform resulted from the discharge machining, and the deform on the material block was measured. The density of energy distribution was different along the kerf, which results in the inhomogeneity deform and ever more frequent breakage of wire. This finding proved the rule which the hole for wire must be drilled before machining must be complied with.
\end{abstract}

\section{Introduction}

Wire Electrical discharge machining (WEDM) has been widely used in various fields since it is capable of achievement of products with a high degree of accuracy and complex shape. Thanks to its multiple parameters and complicated machining mechanism, great efforts are still made to get a better understanding of WEDM since its application in industry[1-2]. Some researchers optimized process parameters to improve efficiency through experiments even if the operators' experience or conservative technological date can be relayed heavily on, which often produced inconsistent machining performance[3-5]. Others have been exploring new application such as dressing the diamond grinding wheel and new materials like advanced ceramics and MMCs by means of WEDM and hybrid WEDM[6-9].

Wire electrode feeds in certain speed to form the predesigned surface profile in at least two directions during WEDM with the help from CNC system, some investigations into control strategy on wire feed has been doing to advance machining quality by avoidance of wire lag and vibration and even breakage while at a higher speed[3]. Researchers have brought about several of control system of wire electrode, for example, fuzzy control system as well as adaptive control system including inaccuracy and self-tuning kinds.[10-11] The complex and random nature in WEDM strongly requires the deterministic and stochastic techniques in order to achieve a more cost efficiency and cost effectiveness of machining operation, finally to unattended operation[12].

Years of continuous efforts by researchers and practitioners in above-mentioned fields of WEDM, has made huge progress in improvement in machining performance. However, the machining efficiency is much more slowly than convention method such as turning and drilling and milling, through the rough and finishing cut has been widely accepted in practice. These results can be fundamentally attributed to little knowledge of thermal effect in WEDM, which can inevitably hinder further progression of WEDM to a large extent. This paper investigates into the thermal effect in WEDM through novel experiment designed, whose findings can contribute to advance the process modeling and develop the new equipment more quickly.

\section{Experimental Setup}

In this study, experiment work was conducted on wire EDM of Mitsubishi FA20M machine, equipped with de-ionized water as the dielectric fluid and brass wire of $0.20 \mathrm{~mm}$ diameters as electrode. Experiment was performed with AISI 1045 carbon structural steel plate in width of 20mm as workpiece, and the machining parameters were automatically gotten from the conservative 
technological date by machine builder. Pulse amplitude and cutting rate were displayed digitally on the machine.

The shape of machined parts chosen was shown in Fig. 1 whose data were gotten from the standard specification for sample preparation for strain test, and Fig. 2 means the motion trajectory of wire electrode designed specially. To investigate the effect of electric energy on wire EDM, the machined parts are firstly large cross- sectional area related data in Fig1, and secondly the through-hone needed commonly on raw plate in wire EDM was not done to cut the parts traditionally from the raw plate, but the start point of wire electrode was chosen out of the plate, so that the surrounding environment of part cut was not closed, then the machining energy released was given out freely.

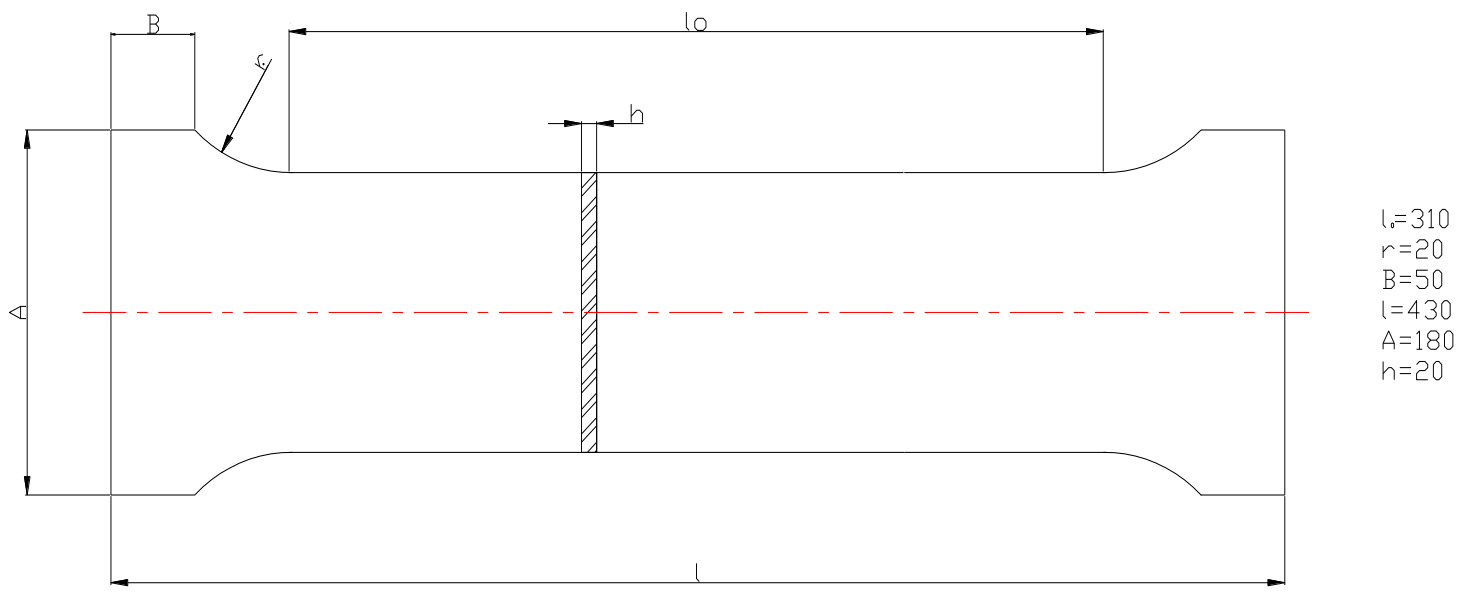

Figure 1. Schematic drawing of the machined part

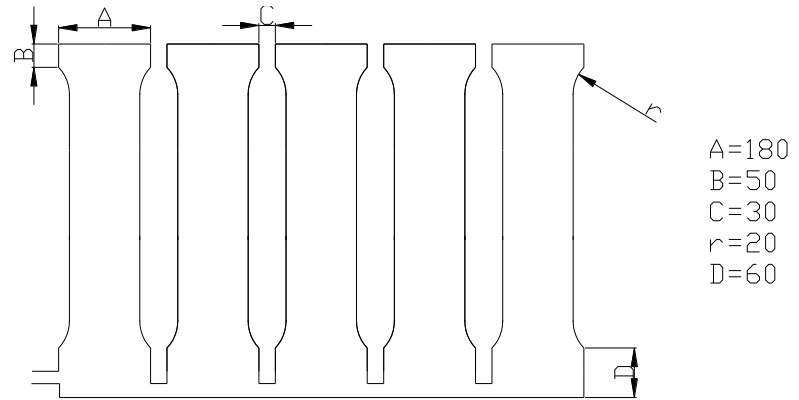

Figure 2. Tool path of electrode wire designed

\section{Results and Discussion}

During the experiment, wire breakage took place for three times, where the position on the way was $564 \mathrm{~mm}, 1112.5 \mathrm{~mm}, 1661 \mathrm{~mm}$ respectively. The above data was measured from the beginning point on the machine parts not the body edge of raw plate. Each time the wire electrode went back to the starting point and moved along the designed way again to finish the cutting process.

The reason for wire breakage in process is naturally attributed to stress level exerted on wire electrode higher than the ultimate strength of material. Higher stress level results from great change of material characterics in processing zone, because the material ahead of wire electrode deform heavily with the kerf after removal materials.

In WEDM through-hole on raw materials was made to the kerf within the space of raw material, especially the cavity shape on parts is required. During the discharge, the shape of energy distribution is radial, then, the nearer the distance from the wire electrode, the greater the energy existence. So the surrounding materials of kerf on raw material can deform homogeneously under the action of discrete 
pulse discharge. At the same time, the de-ionized water full of kerf absorbs the heat from discharge, and flows in the scope of kerf. Under this condition of wire EDM commonly, the closed kerf in the raw material block can prevent discharge energy from spreading out non-uniformly, so that the local stress concentration is not formed in raw material, resulting in the uniform characterics of machined material. One side of materials near kerf belonging to the raw material block can deform with the energy absorption, but deform distribution is thought to be nearly zero because the symmetry of cross-section shape in total space of material block. So the wire EDM continues proceeding until the machined part cut from material block. From the view of entropy, its value climbs up and then declines, which results from the constraints on maximum volume of raw material. Basically speaking, projective closure of kurf on material block brings out the existence of the above constraints.

Once the projective closure of kurf on material block is broken, the constraints on volume of raw material do not take effect on deformity from discharge energy. The entropy increase can bring out plastic deform, the non-uniformity of deform in different parts fabricates the stress concentration. The head of kerf on material block take great change to cut removal material in the form of chips, where the energy density is the maximums and energy loss rate is the minimum for the space volume. Material strengthen in this position increases quickly, while de-ionized water moves strenuously because the chips can be immersed and delayed, which results in the increase in wire temperature , and finally the decrease in wire strength. The jointed action of both aspects come the following things true easily, that is, the stress efforted from environmental objects is greater than the instantaneous material strength, which is much less than the stationary numerical found in the material performance parameter table.

To deeply investigate the deformity of material block from discharge energy, the shapes in remainder of raw plate are chosen to make measurement after one week of finishing experiment. The result and analysis of measurement deform was seen in Fig.3.
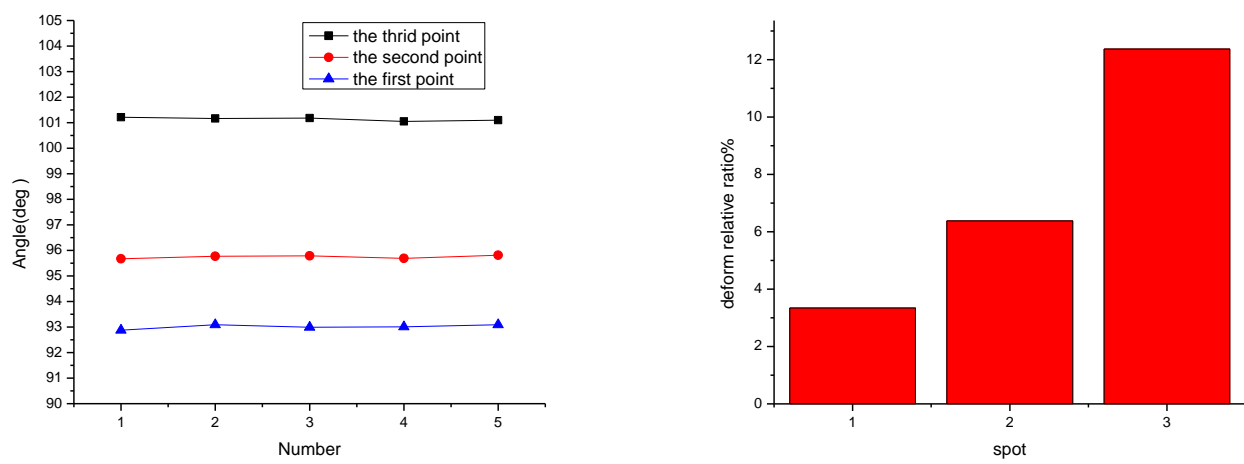

Figure 3. Result and analysis of measurement deform

The designed angle between adjacent segments along the kerf in specification is 90 degrees; the actual angle after machining is between 93 degrees and 101 degrees. The deform relative ratio in the form of percent on the spots was computered. The range of the computer is between $3.35 \%$ and $12.38 \%$.

The cross aera of deform does contribute to the result. The thinner the width, the larger the deformation, which results from the effect of discharge energy and the loss of integrity of raw block. The latter effect takes greater role, which results in the inhomogenity deform along the tool path. The open kerf did not effectively prevent discharge energy from spreading out on raw material block,so the side of mateials near the kerf belonging to the raw material block get the energy flow from the discharge quickly. The energy delivered in discharge on can make surrrounding materials near kerf become deformations, moreover, the deformations on mateials can be accumulated so that the obvious deform in the terms of shape can be found. The results proved the closed kerf must be precondition for wire electrical discharge machining, so the hole for wire must be drilled before fixed 
workpiece in machining. This helped the deform to balance again quickly, althrough the energy disleased for longer time.

\section{Summary}

In this paper, the open kerf was specially designed on the raw block with large cross section area, in order to investigate the role of discharge energy in machining. The findings were followed: the energy delivered in discharge on spread in the total space of materials block, and density of energy distribution was different on raw material block, which results in the inhomogenity deform on the raw material block. The energy delivered in discharge on can make surrounding materials near kerf become deformations, the deformation can be constrained because closed kerf exist in common machining, the deform can be homogeneity quickly. So the rule which the hole for wire must be drilled before machining must be complied with.

\section{Acknowledgements}

The author wishes to acknowledge the support from the principal fund of $\mathrm{Xi}$ 'an technological university (XAGDXJJ-605-010000807).

\section{References}

[1] K. H. Ho, S. T. Newman, S. Rahimifard, R.D. Allen, State of the art in wire electrical discharge machining (WEDM), J. Mach. Tools Manuf. 44 (2004) 1247-1259.

[2] ANISH Kumar, Dr. Vinod kumar, Dr. Jatinder Kumar, A review on the state of the art wire electric discharge machining (WEDM), J. Mech. Eng. Res. Dev. 1(2001)23-39.

[3] Brajesh Kumar Lodhi, Sanjay Agarwal, Optimization of machining parameters in WEDM of AISI D3 steel using Taguchi technique, Procedia CIRP. 14(2014)196-199

[4] J. B. Saedon, Norkamal Jaafar, Mohd Azman Yahaya, NorHayati Saad, Mohd Shahir Kasim, Multi-objective optimization of titanium alloy through orthogonal array and grey relational analysis in WEDM, J. Procedia Tech. 12(2014) 823-840

[5] Bijaya Bijeta Najeta Nayak, Siba Sankar Mahapatra, Optimization of WEDM process parameters using deep cryo-treated inconel 718 as work material, J. Eng. Sci. Tech. 19(2016)161-170

[6] J. A. Sanchez, I. Pombo, I. Cabanes, R. Ortiz, L. N. Lopez de Lacalle. Electrical discharge truing of metal-bonded CBN wheels using single-point electrode, J. Mach. Tools Manuf. 48(2008) 362-370

[7] Eduardo Weingärtner, Raoul Roth, Friedrich Kuster, Marco Boccadoro, Frank Fiebelkoren. Electrical discharge dressing and its influence on metal bonded diamond wheel, J. CIRP Ann. Man. Tech. 61(2012) 183-186

[8] Anand Sharma, Mohinder Pal Garg, Kapil Kumar Goyaal, Prediction of Optimal Conditions for WEDM of Al 6063/ ZrSiO4(p) MMC, Procedia Mat. Sci. 6(2014) 1024-1033.

[9] Takayuki Tani, Yasushi Fukuzawa, Naotake Mohri, Nagao Saito, Masaaki Okada, Machining phenomena in WEDM of insulating ceramics, J. Mat. Proc. Technol. 149(2004)124-128

[10] S. M. Shahruz. Vibration of wires used in electro-discharge machining, J. SOUND VIB.266 (2003) 1109-1116.

[11]S. F. Miller, Chen. C. Kao, A. J. Shih, Jun Qub, Investigation of wire electrical discharge machining of thin cross-sections and compliant mechanisms, J. Mach. Tools Manuf. 45(2005) 1717-1725.

[12]F. Michel, W. Egrfeld, O. Koch, et al: Proc. International Seminar on Precision Engineering and Micro Technollogy, (Aachen, Gwemany, August 9-12, 2000)123-139. 